\title{
Laser vaporization generation of the SiB and SiAl radicals for matrix isolation electron spin resonance studies; comparison with theoretical calculations and assignment of their electronic ground states as $X^{4} \Sigma$
}

\author{
Lon B. Knight, Jr., Allan J. McKinley, and Robert M. Babb \\ Chemistry Department, Furman University, Greenville, South Carolina 29613
}

Michael D. Morse and Caleb A. Arrington

Chemistry Department, University of Utah, Salt Lake City, Utah 84112

(Received 12 January 1993; accepted 29 January 1993)

\begin{abstract}
The first experimental spectroscopic study of the $\mathrm{SiB}$ and SiAl diatomic radicals is reported. Electron spin resonance results indicate that both molecules have $X^{4} \Sigma$ ground electronic states, in agreement with earlier theoretical calculations. The $\mathrm{SiB}$ and $\mathrm{SiAl}$ radicals were generated in neon matrices at $4 \mathrm{~K}$ by trapping the products produced from the pulsed laser vaporization of their alloys. Electronic structure information for these radicals is especially interesting given the utilization of silicon doped materials in semiconductor applications. The observed nuclear hyperfine interactions ( $A$ tensors) for ${ }^{10} \mathrm{~B},{ }^{11} \mathrm{~B}$, and ${ }^{27} \mathrm{Al}$ in these molecular radicals were compared with the results of $a b$ initio configuration-interaction theoretical calculations which were conducted as part of this experimental study. The neon matrix magnetic parameters (MHz) for $\mathrm{Si}{ }^{11} \mathrm{~B}$ are $D=800(2), g_{\|}=2.0014(8), g_{1}=2.0005(4), A_{1}$ $=92.4(5)$, and $A_{\|}=111(2)$. For $\mathrm{Si}^{27} \mathrm{Al}$ the results $(\mathrm{MHz})$ are $D=9710(2), g_{\|}$ $=1.9994(8)$, and $g_{\perp}=1.9978(4),\left|A_{\perp}\right|=10.3(6)$, and $\left|A_{\|}\right|=43.5(8)$.
\end{abstract}

\section{INTRODUCTION}

The $\mathrm{SiB}$ and $\mathrm{SiAl}$ diatomic radicals have been isolated in neon matrices at $4 \mathrm{~K}$ by trapping the products generated from the pulsed laser vaporization of their solid alloys. Detailed electron spin resonance (ESR) investigations provide conclusive evidence that both radicals have $X^{4} \Sigma^{-}$ electronic ground states in agreement with recent theoretical studies. ${ }^{1}$ This is apparently the first spectroscopic study of the $\mathrm{SiB}$ and $\mathrm{SiAl}$ molecules under any experimental conditions. It was found that the zero field splittings ( $D$ tensors) in both molecules were sufficiently small to permit the detection of more than one fine structure transition at the $X$-band microwave frequency employed in these ESR measurements. This fortunate circumstance made possible an unambiguous determination of both the $g$ and $D$ tensors; in addition, the linewidths of these powder type samples were sufficiently small to resolve the nuclear hyperfine interactions ( $A$ tensors) for ${ }^{10} \mathrm{~B},{ }^{11} \mathrm{~B}$, and ${ }^{27} \mathrm{Al}$.

$A b$ initio configuration-interaction (CI) theoretical calculations of the isotropic and dipolar components of the $A$ tensors were conducted as part of this experimental study using the MELDF suite of programs. ${ }^{2}$ Reasonably close agreement between the observed and calculated $A$ values provides electronic structure information on the valence region of these radicals. The three unpaired electrons of the $X^{4} \Sigma$ state occupy $\sigma^{1} \pi^{2}$ type orbitals involving primarily valence " $p$ " electrons on both atoms. However, in the case of $\mathrm{SiB}$ more boron $2 s$ involvement in the $\sigma$ molecular orbit (MO) was found relative to the aluminum $3 s$ orbital in SiAl. Calculated spin densities from a Mulliken spin population analysis (MSPA), projected onto a minimal set of valence type atomic orbitals, were also used to gain electronic structure information.

The electronic structure of the SiB and SiAl molecules is especially interesting because these molecules are isovalent with several other $X^{4} \Sigma$ diatomic cations and neutral radicals containing seven valence electrons which have been recently studied by matrix isolation ESR and theoretical methods. These include $\mathrm{C}_{2}^{+},{ }^{3} \mathrm{Si}_{2}^{+}, \mathrm{Ge}_{2}^{+}, \mathrm{Sn}_{2}^{+}, \mathrm{Pb}_{2}^{+}, 5$ $\mathrm{BC}^{6} \mathrm{AlC}^{7} \mathrm{GaP}^{+}, 8$ and $\mathrm{GaAs}^{+}, 9$ The five valence electron radicals, $\mathrm{YB}^{+}$and $\mathrm{YAl}^{+}$, have been studied by these methods and also found to have $X^{4} \Sigma^{-}$electronic ground states. ${ }^{10}$ Descriptions of the rare gas matrix isolation technique applied to ESR studies and the methods developed for generating and isolating small ion radicals have been presented previously. ${ }^{11-16}$ The use of neon matrices at $4 \mathrm{~K}$ in combination with various ion generation methods such as pulsed laser vaporization, electron bombardment, photoionization at $17 \mathrm{eV}$, and more recently $X$ irradiation ${ }^{17-19}$ has greatly expanded the types of small radicals which can be investigated by ESR and other spectroscopic methods.

The doping of silicon with boron is important in the microelectronics industry for the production of $p$-type semiconductors. Information regarding the vapor phase composition above boron-silicon alloys is significant because of the possible thin film applications of these materials. Chemical vapor deposition (CVD) or molecular beam methods are employed to manufacture epitaxial thin films of silicon. ${ }^{20}$ Apparently the only experimental studies of the diatomic $\mathrm{SiB}$ molecule reported in the literature have been mass spectrometric measurements. ${ }^{21}$ Several high temperature applications of boron-silicon materials include the fabrication of vaporization sources and the formation of protective thermal coatings by CVD. ${ }^{22}$ Mass spectrometric studies of SiAl have determined a dissociation limit $\left(D_{0}\right)$ of $225 \pm 30 \mathrm{~kJ} \mathrm{~mol}^{-1}$, although no spectroscopic data are available for this diatomic radical. ${ }^{23} \mathrm{Nu}$ - 
merous applications of silicon-aluminum alloys include semiconductors and thin film transistors formed by CVD, IR-reflective coatings, and their use as superconductors. The various CVD processes probably involve the SiAl molecule in the vapor phase above the deposition surface, although this has apparently not been demonstrated by direct experimental measurements.

\section{EXPERIMENT}

The matrix isolation cryostat, ESR equipment, and laser vaporization arrangement used in these studies have been described previously. ${ }^{3,4,18,24}$ The boron/silicon and aluminum/silicon alloys or melts were prepared in an electric arc furnace under an argon atmosphere. Prior to heating in the furnace, small chunks of the elemental metals obtained from Alfa were weighed out in a 1:1 molar ratio. These were then melted in the electric arc furnace and allowed to mix thoroughly by convection, then cooled to form the alloy. An additional boron-silicon sample was prepared which contained ${ }^{10} \mathrm{~B}$ enriched to $94 \%$ over its natural abundance of $20 \%$. The $\mathrm{SiB}$ and $\mathrm{SiAl}$ targets were mounted $5 \mathrm{~cm}$ from the copper matrix deposition surface which was maintained at $4 \mathrm{~K}$ by a closed cycle helium refrigerator (APD-304 HS).

Output from a frequency doubled Nd:YAG laser (where YAG denotes yttrium aluminum garnet) operating at $10 \mathrm{~Hz}$ and $10-15 \mathrm{~mJ}$ per pulse was focused to a spot size of approximately $0.5 \mathrm{~mm}$ on the $\mathrm{SiB}$ and SiAl targets. During the vaporization-deposition process the laser beam was moved across the surface to prevent the drilling of deep holes in the target. A typical matrix deposition, employing continuous neon (or argon) flow at a rate of 5 std $\mathrm{cm}^{3} \min ^{-1}$, was conducted for $45 \mathrm{~min}$. Background pressure in the matrix apparatus was approximately $8 \times 10^{-8}$ Torr prior to starting a deposition. A diagram showing the relative locations of the laser target, the matrix deposition surface, and the $X$-band ESR cavity is presented in Fig. 1 of our recent report on the PdB and PdAl radicals which were shown to have $X^{2} \Sigma$ electronic ground states (see Ref. 24).

\section{ANALYSIS OF ESR SPECTRA}

\section{SiB}

The ESR spectrum observed for ${ }^{28} \mathrm{Si}{ }^{11} \mathrm{~B}$ in a neon matrix at $4 \mathrm{~K}$ is shown in Fig. 1. Expanded scale spectra are presented for each of the three perpendicular $\left(\Theta=90^{\circ}\right)$ absorption features assigned as the $\Delta M_{s}=1$ fine structure transitions labeled $X Y_{1}, X Y_{2}$, and $X Y_{3}$ in the conventional manner. ${ }^{11}$ Each fine structure transition is split into a ${ }^{11} \mathrm{~B}$ $(I=3 / 2 ; 80 \%$ natural abundance) quartet of $33 \mathrm{G}$. The ${ }^{10} \mathrm{~B}(I=3 ; 20 \%$ abundance) septet hyperfine lines appear as much weaker absorption features contained within and partially overlapped by the ${ }^{11} \mathrm{~B}$ quartet lines as expected.

Confirmation that these hyperfine splittings result from a single boron atom is shown in Fig. 1 for the $X Y_{3}$ fine structure group for an $\mathrm{Si}^{10} \mathrm{~B}$ sample enriched to $94 \%$ in ${ }^{10} \mathrm{~B}$. The dominant ${ }^{11} \mathrm{~B}$ quartet seen with natural abundance boron now appears as an intense septet centered at

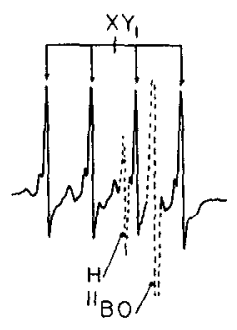

$3088 \quad 3148 \quad 3208$
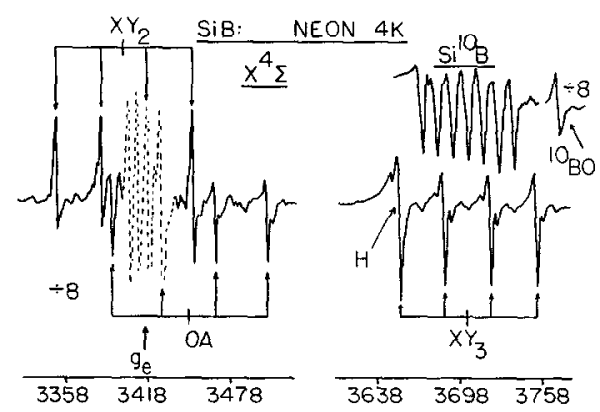

FIG. 1. The observed ESR spectrum for $\operatorname{SiB}\left(X^{4} \Sigma\right)$ isolated in a neon matrix at $4 \mathrm{~K}$ is shown. The three perpendicular $\left(\Theta=90^{\circ}\right)$ fine structure transitions are labeled $X Y_{1}, X Y_{2}$, and $X Y_{3}$, an "extra" or off-angle (OA) absorption feature occurs in the $g_{e}$ magnetic field region; this OA transition is accounted for in the $H_{\text {res }}$ vs $\Theta$ plots shown in Fig. 2. All four of the observed transitions exhibit ${ }^{11} \mathrm{~B}(I=3 / 2)$ quartet hyperfine interaction. With $\mathrm{SiB}$ samples enriched to $94 \%$ in ${ }^{10} \mathrm{~B}(I=3)$, the quartet hyperfine patterns were replaced with ${ }^{10} \mathrm{~B}$ septets as shown for the $X Y_{3}$ fine structure transition centered near 3698 G. ESR signals attributable to other radicals also trapped in the neon matrix under such high energy generation conditions are shown with dashed lines; the $\mathrm{H}$ atom and $\mathrm{BO}$ lines are labeled accordingly, while intense $\mathrm{CH}_{3}, \mathrm{SiH}_{3}$ and boron atom absorptions occur in the $g_{e}$ spectral region.

the same magnetic field position. Similar quartet to septet changes were also observed for the hyperfine structure on the other fine structure transitions and the off-angle (OA) feature. The septet $A$ value is that expected based upon the observed splittings for the ${ }^{11} \mathrm{~B}$ quartet and the known ratio of the nuclear $g_{N}$ factors for ${ }^{10} \mathrm{~B}$ and ${ }^{11} \mathrm{~B}$.

Other ESR lines observed between 0 and $9000 \mathrm{G}$ could be assigned to known radicals such as $\mathrm{Si}_{2}^{+}\left(X^{4} \Sigma\right)$ in the $1700 \mathrm{G}$ region, ${ }^{4} \mathrm{~B}_{2}\left(X^{3} \Sigma\right),{ }^{2510,11} \mathrm{BO},{ }^{26}$ hydrogen and boron atoms, ${ }^{11} \mathrm{H}_{2} \mathrm{O}^{+},{ }^{27} \mathrm{HCO}, \mathrm{SiH}_{3}$, and $\mathrm{CH}_{3}{ }^{11}$ Some of the absorption features associated with these other radicals are indicated in Fig. 1 with dashed lines.

TABLE I. Observed ESR line positions $(G)$ for ${ }^{28} \mathrm{Si}{ }^{11} \mathrm{~B}$ and ${ }^{28} \mathrm{Si}^{27} \mathrm{Al}$ in neon matrices at $4 \mathrm{~K}$ in their $X^{4} \Sigma$ ground electronic states. ${ }^{\text {a }}$

\begin{tabular}{|c|c|c|c|c|c|c|}
\hline & & \multicolumn{2}{|c|}{$X Y_{1}{ }^{b}$} & $X Y_{2}{ }^{b}$ & $\mathrm{OA}^{\mathrm{c}}$ & $X Y_{3}{ }^{b}$ \\
\hline${ }^{28} \mathrm{Si}{ }^{11} \mathrm{~B}$ : & ${ }^{11} \mathrm{~B}\left(M_{I}\right)$ & $\begin{array}{r}3 / 2 \\
1 / 2 \\
-1 / 2 \\
-3 / 2\end{array}$ & $\begin{array}{l}3085 \\
3117 \\
3150 \\
3183\end{array}$ & $\begin{array}{r}3350 \\
3383 \\
(3417)^{d} \\
3450\end{array}$ & $\begin{array}{r}3392 \\
(3430)^{d} \\
3468 \\
3506\end{array}$ & $\begin{array}{l}3654 \\
3686 \\
3720 \\
3754\end{array}$ \\
\hline${ }^{28} \mathrm{Si}^{27} \mathrm{Al}$ & & $1792^{\mathrm{e}}$ & & $6561^{c}$ & $5782^{e}$ & $\mathrm{f}$ \\
\hline
\end{tabular}

${ }^{8}$ The microwave frequency was 9573.1 (3) $\mathrm{MHz}$ for the SiB line positions and $9563.6(3) \mathrm{MHz}$ for SiAl. The calculated line positions, using an exact diagonalization analysis and the magnetic parameters listed in Table II, agree within the experimental uncertainty of $\pm 1 \mathrm{G}$ to these observed line positions.

${ }^{b}$ Following the usual convention, the perpendicular $\left(\theta=90^{\circ}\right)$ fine structure lines $\left(\Delta M_{s}=1\right)$ are denoted $X Y_{1}, X Y_{2}$, and $X Y_{3}$. Each fine structure transition and the $O A$ feature exhibits ${ }^{11} \mathrm{~B}(I=3 / 2)$ quartet hfs.

${ }^{c}$ An intense OA (off-angle) transition was observed at $\theta=42^{\circ}$. See simulated ESR spectrum and $H_{\text {res }}$ vs $\theta$ plots in Fig. 2.

${ }^{d}$ These lines were partially obscured by background absorptions of known impurity radicals in the congested $g_{e}$ magnetic field region.

${ }^{6}$ These $X Y$ features exhibited a small ${ }^{27} \mathrm{Al}(I=5 / 2)$ sextet hfs of $\pm 3.7(3)$ $\mathrm{G}$; the ${ }^{27} \mathrm{Al}$ sextet hfs on the $\mathrm{OA}$ transition at $\theta=29.2^{\circ}$ was $\pm 13.7(4) \mathrm{G}$. 'The $X Y_{3}$ fine structure transition cannot be observed. See Fig. 3. 
TABLE II. Magnetic parameters for ${ }^{28} \mathrm{Si}{ }^{11} \mathrm{~B}$ and ${ }^{28} \mathrm{Si}{ }^{27} \mathrm{Al}$ in their $X^{4} \Sigma$ ground electronic states; results obtained from ESR spectra observed in neon matrices at $4 \mathrm{~K}^{\mathrm{a}}$

\begin{tabular}{llllrrrr}
\hline \hline & $g_{\|}$ & $g_{1}$ & $D$ & \multicolumn{1}{c}{$A_{1}{ }^{b}$} & $A_{\|}{ }^{b}$ & $A_{\text {iso }}{ }^{b}$ & $A_{\text {dip }}{ }^{b}$ \\
\hline${ }^{28} \mathrm{Si}^{11} \mathrm{~B}$ & $2.0014(8)$ & $2.0005(4)$ & $800(2)$ & $92.4(5)$ & $111(2)$ & $99(1)$ & $6(1)$ \\
${ }^{28} \mathrm{Si}^{27} \mathrm{Al}$ & $1.9994(8)$ & $1.9978(4)$ & $9710(2)$ & $-10.3(6)$ & $43.5(8)$ & $8(1)$ & $18(1)$ \\
\hline
\end{tabular}

- $D$ and $A$ values in $\mathrm{MHz}$ where $D$ is the zero field splitting (zfs) parameter and $A$ is the ${ }^{11} \mathrm{~B}$ or ${ }^{27} \mathrm{Al}$ hyperfine interaction. The $A_{\|}$parameters were calculated from the observed hyperfine interaction at $\theta=90^{\circ}$ and the $\mathrm{OA}$ (off-angle) lines at $\theta=42^{\circ}$ for SiB and $\theta=29.2^{\circ}$ for SiAl.

"The absolute signs of the $A$ values for ${ }^{28} \mathrm{Si}^{11} \mathrm{~B}$ could not be obtained from the experimental results alone; however, the positive choices show the best agreement with the MELDF calculations. For ${ }^{28} \mathrm{Si}^{27} \mathrm{Al}$, the experimental results favor the indicated sign choices; however, it should not be considered completely definitive. See text.

All of the observed ESR line positions for $\mathrm{Si}^{11} \mathrm{~B}$ in a neon matrix are listed in Table $I$. The agreement between observed and calculated lines is well within the experimental uncertainty of $\pm 1 \mathrm{G}$. Our previously described computer programs were used to fit the observed lines and determine the magnetic parameters which are listed in Table II. ${ }^{3,13}$ These programs utilize exact diagonalization solutions to the following spin Hamiltonian:

$$
\begin{aligned}
\hat{H}= & \beta \mathbf{H} \cdot \hat{\boldsymbol{g}} \cdot \mathbf{S}+\mathbf{S} \cdot \hat{A} \cdot \mathbf{I}+D\left[S_{z}^{2}-S(S+1) / 3\right] \\
& +Q\left[I_{z}^{2}-I(I+1) / 3\right],
\end{aligned}
$$

where all symbols have their standard meanings. ${ }^{11}$ The inclusion of quadrupole interaction in the Hamiltonian (last term) was not necessary to fit the observed lines within the experimental uncertainty for the $\mathrm{SiB}$ results.

This exact diagonalization routine is also combined with a Lorentzian line-shape program to provide simulated ESR spectra and $H_{\text {res }}$ vs $\Theta$ plots for the various fine structure and nuclear hyperfine transitions. These simulated re-

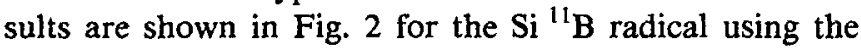
magnetic parameters listed in Table II. The agreement with the experimental spectrum of Fig. 1 is nearly perfect in all respects such as relative phases, line shapes, and relative intensities of the various features. An "extra" or off-angle (OA) absorption feature on the $X Y_{2}$ fine struc-

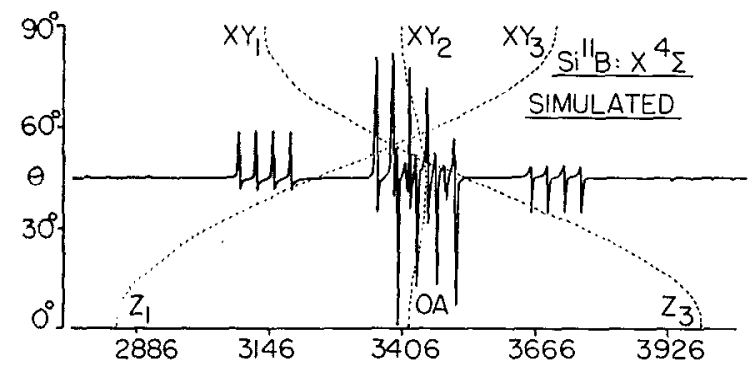

FIG. 2. A simulated ESR spectrum of $\mathrm{Si}^{11} \mathrm{~B}$ in its ${ }^{4} \Sigma$ state is shown using the magnetic parameters listed in Table II; these parameters were obtained from the experimental spectrum of $\mathrm{Si}^{11} \mathrm{~B}$ shown in Fig. 1. The $X Y_{2}$ fine structure transition exhibits an intense off-angle $(O A)$ absorption feature at $\theta=42^{\circ}$. The extremely weak $Z_{1}$ and $Z_{3}$ parallel $\left(\theta=0^{\circ}\right)$ features were not detected in the experimental spectrum. The $H_{\text {res }}$ vs $\theta$ plots for the three $\Delta M_{s}=1$ allowed transitions are shown in dashed lines, where $\theta$ is the angle between the applied magnetic field and the molecular symmetry axis. ture transition is predicted to occur at $\Theta=42^{\circ}$. Of course, the ${ }^{11} \mathrm{~B}$ quartet hyperfine structure on this $\mathrm{OA}$ transition is expected to be different from that observed at $\Theta=90^{\circ}$ on the three $X Y$ lines. This OA feature is clearly evident on the high field side of the $X Y_{2}$ group near the congested $g_{e}$ magnetic field region in the experimental spectrum of Fig. 1. The experimental observation of the ${ }^{11} B$ hyperfine structure on this $O A$ line is most significant since it makes possible the determination of both the $A_{1}$ and $A_{\|}$hyperfine interaction parameters for boron. The absolute signs of the boron $A$ values in SiB could not be determined from these experimental results alone. However, their relatively large magnitude and close agreement with the MELDF calculations indicate that both $A_{\perp}$ and $A_{\|}$are positive.

Direct observation of the parallel $\left(\Theta=0^{\circ}\right)$ transitions in $\mathrm{SiB}$ was not possible given their extremely small intensity as shown in the simulated spectrum of Fig. 2. Based upon previous studies of high spin powder samples, detection of the weaker parallel lines is usually not achieved because of the highly anisotropic nature of the $D$ tensor. ${ }^{8,11}$

Several attempts were made to observe the much weaker ${ }^{29} \mathrm{Si}(I=1 / 2$; natural abundance $4.7 \%)$ doublet hyperfine structure in $\mathrm{SiB}$. Ideally, the individual ${ }^{29} \mathrm{Si} X Y$ lines should have an intensity about 40 times less than that observed for the same transition in the ${ }^{28} \mathrm{Si}^{11} \mathrm{~B}$ radical. While intensity alone may have prevented detection of these features, it is also possible that the ${ }^{29} \mathrm{Si} A_{\perp}$ parameter is less than $6 \mathrm{G}$. If $A_{\downarrow}$ were this small, the weaker ${ }^{29} \mathrm{Si}$ lines would be even more difficult to detect since they would overlap the more intense ${ }^{28} \mathrm{Si}$ central line. The theoretical results presented below do predict quite small ${ }^{29} \mathrm{Si}$ hyperfine interactions for both $A_{\text {iso }}$ and $A_{\text {dip }}$.

\section{SiAl}

An analysis of the observed ESR spectrum assigned to the SiAl radical in its $X^{4} \Sigma$ ground state can best be described by first referring to the simulated spectrum and superimposed $H_{\text {res }}$ vs $\Theta$ plots shown in Fig. 3. (Of course, the detailed analysis had to be conducted before these plots could be generated.) The SiAl radical is a rare and fascinating case for a ${ }^{4} \Sigma$ radical since its zero field splitting ( $D$ value) is intermediate between two extremes. For $D$ large compared to the microwave energy $(h v)$ employed, only one $X Y$ (perpendicular) fine structure transition $\left(M_{s}=1\right.$ ) $2<-M_{s}=-1 / 2$ ) will be detected and it will occur in the 


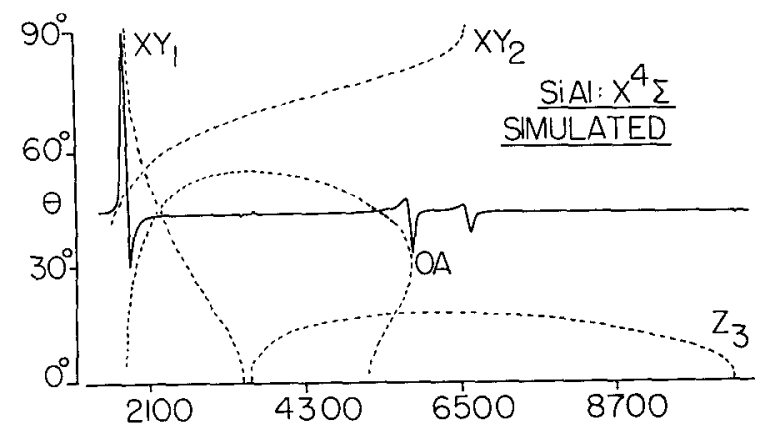

FIG. 3. A simulated ESR spectrum and $H_{\text {res }}$ vs $\Theta$ plots (dashed lines) are shown for SiAl in its $X^{4} \Sigma$ state, using the magnetic parameters listed in Table II. The small ${ }^{27} \mathrm{Al}(I=5 / 2)$ hyperfine structure is not included in this overall presentation. The magnetic energy levels and associated $\Delta M_{S}=1$ transitions are more complex for this case relative to $\mathrm{SiB}$ since the $D$ tensor (zero field splitting) is comparable in magnitude to the microwave energy employed in the ESR measurements. The observed absorption features $\left[X Y_{1}, X Y_{2}\right.$, and the off-angle $(O A)$ line at $\left.\Theta=29^{\circ}\right]$ are shown in Figs. 4 and 5. All three of these observed transitions exhibit $\mathrm{Al}(I=5 / 2)$ hyperfine structure. Our exact diagonalization program calculates the transition probability (TP) at each $\Theta$ and $H$ point and weights the simulated spectrum accordingly. If the calculated TP is below some minimum input parameter, the $H_{\text {res }}$ vs $\theta$ plot abruptly terminates. This is seen to occur for the $X Y_{2}$ fine structure transition below $\Theta \approx 40^{\circ}$.

$g=4$ magnetic field region with a very weak $Z$ or parallel line near $g_{e}$. A detailed description and simulated spectrum for this case have been presented earlier. ${ }^{8-11}$ For $D$ small relative to $h v$, the ${ }^{4} \Sigma$ case will resemble that shown in Fig. 2 as discussed above for the $\mathrm{SiB}$ radical, where all three fine structure transitions can be observed. For SiAl it was found that $D=9710 \mathrm{MHz}$, which is fortuitously close to the microwave frequency of $9564 \mathrm{MHz}$. For such an intermediate case, it is necessary to conduct an exact diagonalization of the spin Hamiltonian and search for off-angle transitions which can be more intense than those at $\theta=90^{\circ}$.

As seen in the simulated spectrum for $\mathrm{SiAl}$ in Fig. 3, the $X Y_{1}$ fine structure transition is predicted to occur near $1800 \mathrm{G}$ with a much weaker $X Y_{2}$ transition near $6500 \mathrm{G}$. An off-angle transition of greater intensity than the $X Y_{2}$ line occurs near $5800 \mathrm{G}$. The $X Y_{3}$ transition does not exist in this case and all five of the $Z$ lines are extremely weak and could not be detected. Our diagonalization program not only computes line positions at each value of $\Theta$ for a given input set of magnetic parameters, it also calculates the transition probability at each $\Theta$ and $H$ point and weights the simulated spectrum accordingly. The input file contains a parameter that sets the minimum transition probability that should be considered. For example, the $H_{\text {res }}$ vs $\Theta$ plot for the transition ending at $X Y_{2}$ breaks off at $\Theta \approx 40^{\circ}$ because the calculated transition probability at that point has fallen below this specified minimum level.

Observation of the $X Y_{1}, X Y_{2}$, and $O A$ transitions and the aluminum $(I=5 / 2)$ sextet hyperfine structure that each one of these major absorption features exhibits provides sufficient information to obtain a unique set of magnetic parameters, namely $g_{1}, g_{\|}, D$, and the aluminum hyperfine tensor components, $A_{\|}$and $A_{1}$. The ESR line

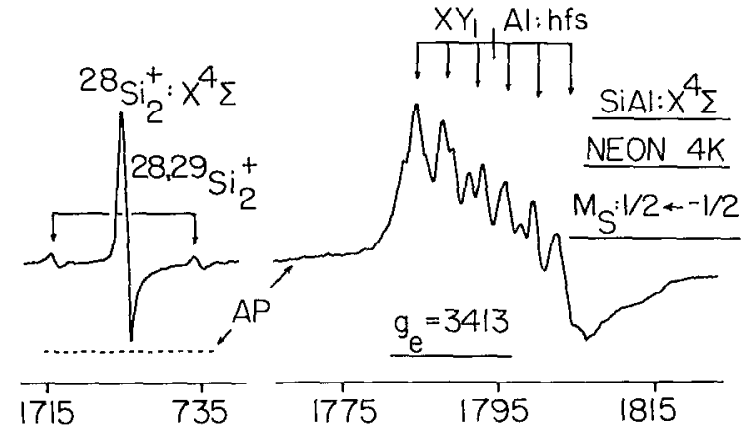

FIG. 4. An expanded scale presentation of the $X Y_{1}$ transition in SiAl exhibiting $\mathrm{Al}(I=5 / 2) \mathrm{hfs}$ is shown for a neon sample at $4 \mathrm{~K}$. The small deviation of this pattern from six equally spaced lines is attributed to quadrupole effects. See the text. Also shown is the ESR spectrum of ${ }^{28} \mathrm{Si}_{2}^{+}$ $\left(X^{4} \Sigma\right)$ and the ${ }^{29} \mathrm{Si}(I=1 / 2)$ hyperfine doublet in ${ }^{28,29} \mathrm{Si}_{2}^{+}$. The assignment of this radical ion species, which is also generated under these laser vaporization conditions, has been previously reported (Ref. 4). The striking contrast between the response of isolated ion radicals and most neutral radicals to the effect of visible light photolysis is shown by the ESR scans labeled AP (after photolysis). The $\mathrm{Si}_{2}^{+}$lines were eliminated while those features belonging to the neutral SiAl radical were unaffected.

positions for $\mathrm{SiAl}$ are listed in Table I with the derived magnetic parameters given in Table II. The exact diagonalization line fitting procedure yielded calculated line positions which agreed with the observed lines within the experimental uncertainty of $\pm 1 \mathrm{G}$. An expanded scale presentation of the $X Y_{1}$ fine structure transition centered at $1792 \mathrm{G}$ exhibiting aluminum sextet hfs of $3.7(2) \mathrm{G}$ is shown in Fig. 4. Figure 5 shows the Al hfs of 13.7(4) G on the OA $\left(\Theta=29.2^{\circ}\right)$ feature centered at $5782 \mathrm{G}$; the $X Y_{2}$ transition centered at $6561 \mathrm{G}$ exhibits Al hfs of 3.6(4) G. Approximately $25 \%$ of the $\mathrm{SiAl}$ radicals were trapped in an alternate site in the neon lattice which is labeled "SITE" in Fig. 5. This minor site had the same Al hyperfine splitting within the indicated experimental uncertainty but its

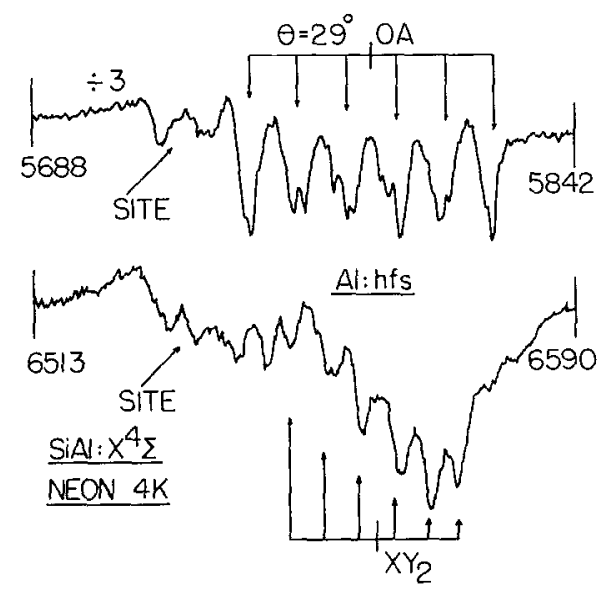

FIG. 5. The $\mathrm{Al}(I=5 / 2)$ hfs of the $X Y_{2}$ and the off-angle (OA) fine structure absorptions are presented on these expanded scale ESR spectra for SiAl in neon at $4 \mathrm{~K}$. See Fig. 3 for the overall simulated spectrum of SiAl in its $X^{4} \Sigma$ ground state. The weaker features labeled "SITE" on the low field side of each spectrum results from an alternate trapping site for $\mathrm{SiAl}$ in the neon lattice. See the text. 


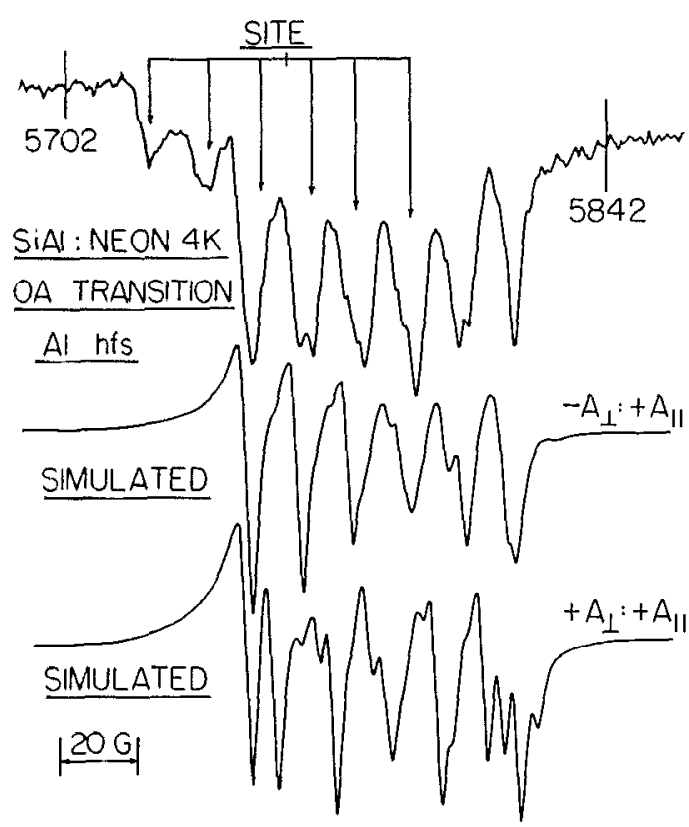

FIG. 6. The observed (top spectrum) off-angle (OA) ESR transition of SiAl, exhibiting an AI $(I=5 / 2)$ sextet hyperfine pattern, is compared with simulated spectra having different sign choices for the aluminum $A_{1}$ and $A_{\|}$parameters. The choice of $A_{1}<0$ and $A_{\|}>0$ shows the best qualitative agreement with the observed spectrum compared to simulations for the other three possible sign combinations. The choice of $A_{1}>0$ and $A_{1}>0$ is shown in the bottom spectrum. The aluminum sextet resulting from SiAl trapped in an alternate site in the neon lattice with a slightly smaller $D$ value is labeled "SITE." See the text.

$D$ value was $45 \mathrm{MHz}$ less than that of the major trapping site whose $D$ value was $9710 \mathrm{MHz}$. This small difference in $D(0.5 \%)$ causes a much larger "site shift" for the high field $\mathrm{OA}$ and $X Y_{2}$ absorption features relative to the low field $X Y_{1}$ transition. An analysis shows that the site shift for $X Y_{2}$ is $12.5 \mathrm{G}$ while it is only $0.8 \mathrm{G}$ for the low field $X Y_{1}$ transition. Typically, the $D$ tensor is more sensitive to local environmental effects than the $g$ and $A$ tensors. ${ }^{11}$

As discussed below, the absolute signs of the $A_{1}$ and $A_{\|}$parameters for aluminum influence the experimental values of $A_{\text {iso }}$ and $A_{\text {dip }}$ since $A_{\text {iso }}=\left(A_{\|}+2 A_{\perp}\right) / 3$ and $A_{\text {dip }}$ $=\left(A_{\|}-A_{1}\right) / 3$. When ESR spectra of powder type samples yield only the parallel $\left(\Theta=0^{\circ}\right)$ and perpendicular $(\Theta$ $=90^{\circ}$ ) line positions, it is not usually possible to determine the absolute signs of $A_{1}$ and $A_{\|}$for a given magnetic nucleus. However, in some cases an exact diagonalization analysis allows for the inclusion of complex terms and absolute signs in off-diagonal positions, and the absolute signs of the $A$ values can be determined if nuclear hyperfine structure is resolved on off-angle (OA) absorption features. Since our exact diagonalization analysis routines incorporate this feature, being built on Iwasaki's thorough derivation, ${ }^{28}$ it is possible to generate simulated spectra for the off-angle feature of SiAl which is sensitive to the absolute signs of the aluminum $A_{1}$ and $A_{\|}$input parameters. Considerable effort was spent in comparing the obscrved $\mathrm{OA}$ aluminum sextet with simulated spectra that involved all four sign choices for $A_{1}$ and $A_{\|}$. As shown in Fig. 6, simulated spectra for the two cases of $A_{\perp}<0, A_{\|}>0$, and
$A_{\perp}, A_{\|}>0$ are compared with the observed $\mathrm{OA}$ aluminum hfs which occurs at $\Theta=29.2^{\circ}$. Clearly, the case of negative $A_{\perp}$ and positive $A_{\|}$input parameters yields closer agreement with the observed spectra. The four lowest field hyperfine lines are somewhat distorted by overlap from SiAl signals in the alternate trapping site; however, the two highest field lines of the $\mathrm{Al}$ sextet pattern are free of such complications. The other two sign combinations (not shown) produced noticeably poorer agreement with experiment. Numerous simulations were conducted with different linewidths and with a range of aluminum quadrupole coupling constants - a parameter that was independently determined from an analysis of the $X Y_{1}$ transition as discussed below. Experimental spectra from five independent neon matrix samples deposited under different experimental conditions were examined for consistency in evaluating the simulated vs experimental comparisons. In summary, the comparison with simulated spectra for the OA absorption for SiAl indicates a strong preference in the sign choices of $A_{1}<0$ and $A_{\|}>0$. However, this sign conclusion should be considered as a probable rather than a certain experimental finding given the powder nature of the sample. In fact, having both $A$ values positive causes the experimental $A_{\text {dip }}$ value to show closer agreement with the MELDF calculated value as discussed in a later section.

The Al sextet hyperfine structure of the $X Y_{1}$ fine structure transition exhibited slight deviations from an equally spaced six-line hyperfine pattern. The individual spacings varied between 3 and $4 \mathrm{G}$, just outside the experimental uncertainty, and a few extra shoulders were present on some lines. After allowing for possible site shift effects discussed earlier, aluminum quadrupole complications were found to be responsible for these properties and comparisons with simulated spectra yielded a quadrupole coupling constant $(\bar{Q}=e q Q)$ in the range of $32-44 \mathrm{MHz}$. It is reasonable that quadrupole effects could be present since the Al $A_{\perp}$ parameter is only $3.7 \mathrm{G}$. For the OA line with $A=13.7 \mathrm{G}$, the introduction of quadrupole interaction in this range did not show as large effects on the simulated spectra. Second-order perturbation calculations show that quadrupole induced shifts of the allowed hyperfine lines depend upon $\bar{Q}^{2} / A .^{11}$

\section{ELECTRONIC STRUCTURE}

The general electronic structure of the main group diatomic silicides, $\mathrm{MSi}$, has been recently discussed by Boldyrev and Simons. ${ }^{1}$ These investigators have found that both $\mathrm{SiB}$ and $\mathrm{SiAl}$ adopt the ground electronic configuration $\sigma^{1} \pi^{2}{ }^{4} \Sigma^{-}$, as we have now experimentally confirmed. This result is somewhat surprising, since one might naively expect a two-electron $\sigma$ bond to be favored when a $p^{1}$ atom such as boron or aluminum approaches a $p^{2}$ atom such as silicon, resulting in a ground state described as $\sigma^{2} \pi^{1},{ }^{2} \Pi_{r}$ Indeed, other one-electron radicals such as $\mathrm{H}, \mathrm{F}, \mathrm{Cl}, \mathrm{Br}$, and $\mathrm{I}$ all bond to silicon by forming a two-electron $\sigma$ bond, leading to ground states of $X^{2} \Pi_{r}{ }^{29}$ The essential difference between these one-electron radicals $(\mathrm{H}, \mathrm{F}, \mathrm{Cl}, \mathrm{Br}$, and $\mathrm{I}$ ) and boron or aluminum, as identified by Boldyrev and Simons, is that boron and aluminum are electropositive, 
instead of electronegative. Other electropositive elements, such as $\mathrm{Li}$ and $\mathrm{Na}$, were also calculated to combine with silicon to give $\sigma^{1} \pi^{2},{ }^{4} \Sigma^{-}$ground states, consistent with the idea that the electronegativity of the element bonding to silicon was the factor which determined the ground state.

A different way of considering the bonding in these electropositive compounds of silicon is to consider the atoms coming together as $\mathrm{M}^{+}+\mathrm{Si}^{-}$, rather than as neutrals. This ion-pair limit lies rather low in energy in electropositive compound such as $\mathrm{BSi}, \mathrm{AlSi}, \mathrm{LiSi}$, and $\mathrm{NaSi}$, lying only $6.911,4.601,4.005$, and $3.753 \mathrm{eV}$ above ground state atoms, respectively. Moreover, the strongly attractive Coulomb interaction between the $\mathrm{M}^{+}$and $\mathrm{Si}^{-}$ions makes the ion-pair state drop quickly in energy as the atoms are brought together, bringing the ion-pair state even closer to the valence states of the molecule. As a result, it is possible that the ion-pair state may actually become a predominant contributor to the ground state in these electropositive species. In this regard, we may note that the ground state of the $\mathrm{Si}^{-}$anion is $p^{3},{ }^{4} S^{0}$, which can combine with the ground state of $\mathrm{B}^{+}\left({ }^{1} S_{0}\right), \mathrm{Al}^{+}\left({ }^{1} \mathrm{~S}_{0}\right), \mathrm{Li}^{+}\left({ }^{1} S_{0}\right)$, or $\mathrm{Na}^{+}\left({ }^{1} S_{0}\right)$ to give only a ${ }^{4} \Sigma$ term. The first excited state of $\mathrm{Si}^{-}\left(p^{3},{ }^{2} D^{0}\right)$ lies $0.862 \mathrm{eV}$ above the ${ }^{4} S^{0}$ ground state, ${ }^{30}$ thereby diluting its influence considerably, so that it may be ignored in the first approximation. With these considerations in mind, it is perhaps not so surprising that the ground states of $\mathrm{LiSi}$, $\mathrm{NaSi}, \mathrm{BSi}$, and $\mathrm{NaSi}$ are calculated to be ${ }^{4} \Sigma^{-}$. Indeed, even if the ${ }^{1} S+{ }^{4} S^{0}$ ion-pair state is not the primary contributor to the ground state of the molecule, it will nevertheless cause a significant configuration interaction with the covalent ${ }^{4} \Sigma^{-}$state which arises from the ground separated atom limit of ${ }^{2} P^{0}$ (or ${ }^{2} S$ in the case of $\mathrm{Li}$ or $\mathrm{Na}$ ) $+\mathrm{Si}\left(3 p^{2},{ }^{3} P\right)$, leading to a preferential stabilization of the ${ }^{4} \Sigma^{-}$state.

\section{$\mathrm{SiB}$}

Information concerning the valence electronic structure of $\mathrm{SiB}$ can be obtained from the observed and calculated $A_{\text {iso }}$ and $A_{\text {dip }}$ nuclear hyperfine parameters defined in the following manner:

$$
\begin{aligned}
& A_{\text {iso }}=\frac{8}{3} \pi g_{e} g_{n} \beta \beta_{n}\langle\delta(r)\rangle, \\
& A_{\text {dip }}=\frac{1}{2} g_{e} g_{n} \beta_{d} \beta_{n}\left\langle\left(3 \cos ^{2} \theta-1\right) / r^{3}\right\rangle,
\end{aligned}
$$

where all symbols have their standard meanings and the averages are taken over the spin density. ${ }^{11}$ The $A$ values were calculated for ${ }^{29} \mathrm{Si}$ and ${ }^{11} \mathrm{~B}$ using the MELDF suite of programs $^{2}$ at the equilibrium bond distance of $1.905 \AA$ previously determined by Boldyrev and Simons for the $X^{4} \Sigma$ ground state. ${ }^{1}$ Our hyperfine calculations were conducted at the multireference single and double configuration interaction (MRSDCI) level using the double zeta plus polarization (DZP) Dunning basis set, employing 342719 spin adapted configurations and 40 reference configurations which were selected by the magnitudes of the CI coefficients. ${ }^{31}$ The CI MELDF calculation, which includes all single excitations and double excitations selected by perturbation theory, yielded a ground state energy of
-313.6492 a.u. The perturbation energy threshold employed in the hyperfine calculation was $E_{T}=1 \times 10^{-8}$ a.u.

For the $\mathrm{SiB}$ radical, the calculated ${ }^{29} \mathrm{Si}$ hyperfine parameters were $A_{\text {iso }}=14$ and $A_{\text {dip }}=4.5 \mathrm{MHz}$; for ${ }^{11} \mathrm{~B}, A_{\text {iso }}$ $=105$ and $A_{\text {dip }}=5.6 \mathrm{MHz}$. Assuming that the $A_{\perp}$ and $A_{\|}$ parameters determined from the ESR results for ${ }^{11} \mathrm{~B}$ are positive, the experimental values of $A_{\text {iso }}=99(1)$ and $A_{\text {dip }}$ $=6$ (1) $\mathrm{MHz}$ show excellent agreement with the MELDF calculated results. Unfortunately, the ${ }^{29} \mathrm{Si}$ hyperfine lines were too weak to be observed given the low natural abundance of this isotope. The additional anisotropy that the ${ }^{29} \mathrm{Si}$ hfs would be expected to introduce would make detection of these weak satellite features even more difficult.

The small calculated $A_{\text {iso }}$ value for ${ }^{29} \mathrm{Si}$ in $\mathrm{SiB}$ relative to the calculated free atom parameter for a silicon $3 s$ electron of $-4600 \mathrm{MHz}$ (Ref. 32) indicates practically no $3 s-3 p_{z}$ hybridization or $3 s$ participation in the bonding MO's. However, for boron the $2 s-2 p_{z}$ hybridization is considerably larger. The boron $2 s$ spin density in $\mathrm{SiB}$, using the free atom $A_{\text {iso }}$ parameter of $2547 \mathrm{MHz}^{32}$ can be estimated by the expression $3 A_{\text {iso }}$ (molecule) $/ A_{\text {iso }}$ (atom) where the factor of 3 is required to account for the spin dilution effects in this ${ }^{4} \Sigma$ radical. This commonly applied free atom comparison method (FACM) yields a boron $2 s$ character of 0.12 . The observed boron dipolar interaction cannot be converted to $2 p_{z}$ character in the standard manner as that employed in ${ }^{2} \Sigma$ radicals since the $2 p_{x}$ and $2 p_{y}$ orbitals also influence $A_{\mathrm{dip}}$ in this ${ }^{4} \Sigma$ radical. In fact, equal participation of the three $2 p$ orbitals would produce a boron dipolar interaction of zero, neglecting the small effects of spin density located on the silicon atom. Hence the three unpaired electrons in $\mathrm{SiB}$ occupy the $\sigma^{1} \pi^{2}$ bonding orbitals where the $\sigma \mathrm{MO}$ is predominantly a linear combination of $2 s$ and $2 p_{z}$ boron orbitals and the $3 p_{z}$ orbital of silicon. The singly occupied $\pi_{x}$ and $\pi_{y}$ MO's contain the $2 p_{x}$ and $2 p_{y}$ orbitals of boron and the $3 p_{x}$ and $3 p_{y}$ orbitals of silicon.

The MELDF suite of programs can project the total CI wave function onto a minimal set of valence atomic orbitals, yielding the following gross Mulliken spin population analysis (MSPA) results for the ground ${ }^{4} \Sigma$ state of SiB. B: $2 s=0.076,2 p_{y}=0.19$, and $2 p_{x}=2 p_{y}=0.15$; and for Si: $3 s$ $=0.016,3 p_{z}=0.052$, and $3 p_{x}=3 p_{y}=0.18$ where the total spin density has been normalized to unity. A discussion of this method has been presented in our earlier work on the $\mathrm{SiO}^{+}$radical cation. ${ }^{33}$ This MSPA result for the boron $2 s$ character compares reasonably well with the FACM estimate of 0.12 .

In addition, these calculated MSPA results can be used to predict the boron $A_{\text {dip }}$ parameter in $\mathrm{SiB}$ provided the spin density on silicon is neglected. This is reasonable approximation given the large bond distance involved and the $r^{-3}$ dependence for the dipolar interaction. It can be shown that the $A_{\text {dip }}$ parameter for boron in $\mathrm{SiB}$ is approximately $\left(C_{2 p z}^{2}-C_{2 p x}^{2}\right) \bar{A}_{\text {dip }}$, where $\bar{A}_{\text {dip }}=65 \mathrm{MHz}$ is the dipolar parameter for a boron atom and the orbital characters $\left(C_{i}^{2}\right)$ are provided by the MSPA results cited above. The $A_{\text {dip }}$ parameter for boron calculated in this manner has a value of approximately $3 \mathrm{MHz}$ compared to the experimental result of 6(1) MHz. The inclusion of spin density centered 
on silicon would increase this crude estimate of $A_{\text {dip }}$, causing it to show closer agreement with the experimental result.

\section{SIAI}

MELDF hyperfine calculation for SiAl employing the DZP Dunning basis set at the theoretical bond distance of $2.430 \AA$ previously determined by Boldyrev and Simons ${ }^{1}$ yielded $A_{\text {iso }}=0.4$ and $A_{\text {dip }}=-0.9 \mathrm{MHz}$ for ${ }^{29} \mathrm{Si}$ and $A_{\text {iso }}$ $=1.2$ and $A_{\text {dip }}=9.7 \mathrm{MHz}$ for ${ }^{27} \mathrm{Al}$. Forty reference configurations were employed in the final CI wave function which contained 689978 spin adapted configurations yielding a ground state $\left(X^{4} \Sigma\right)$ energy of -531.0438 a.u. The threshold selection energy $\left(E_{T}\right)$ was set at $1 \times 10^{-8}$ a.u. The ${ }^{29} \mathrm{Si}$ hfs was not observed in the experimental ESR spectrum of SiAl because of its low intensity and its apparent close proximity to the intense ${ }^{28} \mathrm{Si}^{27} \mathrm{Al}$ lines which would be expected based upon the extremely small magnitude of the calculated ${ }^{29} \mathrm{Si} A$ values.

The ${ }^{27} \mathrm{Al}$ hfs was resolved into its $A_{\perp}$ and $A_{\|}$components by utilizing the OA hyperfine splitting at $\Theta=29^{\circ}$, as described in the spectral analysis section above. The most reasonable sign choices are $A_{\perp}= \pm 10.3(6)$ and $A_{\|}$ $=+44(2) \mathrm{MHz}$ where the simulated spectral comparisons with the observed OA feature clearly favors the negative choice for $A_{1}$. (See Fig. 6 and the earlier discussions.) With both experimental $A$ parameters positive, we obtain $A_{\text {iso }}=22$ (1) and $A_{\text {dip }}=11(1) \mathrm{MHz}$. This positive choice for $A_{\downarrow}$ produces close agreement with the calculated $A_{\text {dip }}$ result of $9.7 \mathrm{MHz}$ for aluminum and poor agreement with the calculated $A_{\text {iso }}$ value of $1.2 \mathrm{MHz}$. Using the negative choice for $A_{1}$, we obtain $A_{\text {iso }}=8(1)$ and $A_{\text {dip }}=18(1)$ $\mathrm{MHz}$. While the negative $A_{\perp}$ choice improves the agreement between theory and experiment for $A_{\text {iso }}$, it causes the experimental $\boldsymbol{A}_{\text {dip }}$ parameter to exceed the theoretical prediction by almost a factor of 2 . It is not surprising that the theoretical calculations produce poor quantitative agreement with experiment for the isotopic hyperfine interaction when its magnitude is inherently small. The $A_{\text {iso }}$ calculation involves the difference between two large contributions of opposite sign, namely the contributions made by the core electrons and the valence electrons. The delicate balancing of these two large contributions to achieve accurate $A_{\text {iso }}$ values is especially difficult in such cases. ${ }^{33}$ However, such theoretical calculations typically predict $A_{\text {dip }}$ parameters within $10 \%-20 \%$ of the observed, as was the case with the SiB example. However, such comparisons with experimental results have usually involved the lighter firstrow atoms.

The sensitivity of the calculated $\mathrm{Al}$ hyperfine parameters to the SiAl bond distance employed was tested by repeating the calculations at $\pm 0.1 \AA$ from the calculated $r_{\text {eq }}$ value of $2.430 \AA$. The shorter bond length caused the aluminum $A_{\text {iso }}$ parameter to increase from 1.2 to $2.7 \mathrm{MHz}$ while use of the longer bond length caused a sign reversal in $A_{\text {iso }}$ to $-1.2 \mathrm{MHz}$. The $A_{\text {dip }}$ paramcter increased from 9.7 to $10.3 \mathrm{MHz}$ when the bond length was reduced by 0.1 $\AA$ and decreased to $9.2 \mathrm{MHz}$. when the length was increased by $0.1 \AA$ A.
With either sign choice for the aluminum $A_{\perp}$ parameter, it is clear that the aluminum $A_{\text {iso }}$ parameter is relatively much smaller than its boron counterpart in SiB. The atomic $A_{\text {iso }}$ parameter for an aluminum $3 s$ electron is 3911 MHz. ${ }^{32}$ The small $A_{\text {iso }}$ value for Al implies significantly less $3 s-3 p_{z}$ hybridization on aluminum in the singly occupied $\sigma$ type MO in SiAl. Decreasing $s-p$ hybridization for the heavier atoms among the representative elements is the expected behavior. The considerably lower ionization energy of aluminum ( $5.98 \mathrm{eV}$ ) compared to boron $(8.30 \mathrm{eV})$ should increase the ionic character of SiAl and cause a shift of the total spin density to silicon. This qualitative prediction is consistent with the following calculated MSPA orbital occupancies for SiAl which can be compared with the results given above for $\mathrm{SiB}$. Al: $3 s=0.018$, $3 p_{z}=0.10,3 p_{x}=3 p_{y}=0.076 ;$ and Si: $3 s=0.020,3 p_{z}=0.19$, $3 p_{x}=3 p_{y}=0.25$. The $2 p_{z} / 2 s$ ratio on boron in $\mathrm{SiB}$ is 2.4 compared to 5.6 for the $3 p_{z} / 3 s$ ratio on aluminum in SiAl. The spin population in each of the three $2 p$ orbitals on boron is approximately twice as large as that for the three $3 p$ orbitals on aluminum. The boron $2 s$ population is 4.3 times as large as the aluminum $3 s$ population. Overall, the total valence spin density on boron is 0.56 compared to 0.27 on aluminum. According to GAUSSIAN 92 calculations at the second-order M $\varnothing$ ller-Plesset perturbation theory (MP2) level, changes in the total charge distributions also seem consistent with these overall spin density shifts. Silicon has a net charge of +0.16 in $\mathrm{SiB}$ and -0.15 in SiAl.

The MELDF calculated quadrupole coupling constant (eqQ) is quite small for boron in $\mathrm{Si}^{11} \mathrm{~B}$, having a value of only $-2.3 \mathrm{MHz}$. Given the relatively large magnitude of the hyperfine interaction in $\mathrm{Si}^{11} \mathrm{~B}$, no quadrupole effects on its ESR spectrum were observed or expected. Spectral simulations revealed that increasing the calculated value by a large amount by a large amount (X5) would still not be sufficient to influence the spectrum. However, the calculated value of $e q Q=-24 \mathrm{MHz}$ for aluminum in $\mathrm{SiAl}$ was much larger and would be expected to influence the ESR spectrum, especially given the small hyperfine parameter for aluminum. As discussed above, we found through spectral simulation comparisons that $|e q Q|$ is most probably in the range of $32-44 \mathrm{MHz}$. It is interesting that both the theoretical calculation of $A_{\text {dip }}$ and $e q Q$ for aluminum are noticeably below the experimental results. Even though $e q Q$ depends on the total field gradient, the quadrupole coupling constant and $A_{\text {dip }}$ would increase if there was more $3 p_{z}$ character in the singly occupied $\sigma \mathrm{MO}$ of SiAl. More extensive calculations at higher levels of theory employing a larger basis set are required to address these properties of the SiAl molecule.

The larger value of $\Delta g_{\perp}\left(\Delta g_{\perp}=g_{e}-g_{\perp}\right)$ for SiAl of 0.0045 (4) compared to $\Delta g_{1}=0.0018(4)$ for $\mathrm{SiB}$ may reflect the larger atomic spin-orbit parameter of $75 \mathrm{~cm}^{-1}$ for aluminum relative to just $11 \mathrm{~cm}^{-1}$ for boron. However, we cannot be certain about the significance of the $\mathrm{B}-\mathrm{Al}$ influence since the large silicon spin-orbit parameter of 149 $\mathrm{cm}^{-1}$ could cause even small electronic structure changes involving silicon orbitals to dominate the $g$ shift. The theoretical calculations of Boldyrev and Simons indicate a 
low-lying excited ${ }^{2} \Pi$ state at approximately $8000 \mathrm{~cm}^{-1}$ for $\mathrm{SiB}$ and $11000 \mathrm{~cm}^{-1}$ for SiAl above their ground $X^{4} \Sigma$ states. It can be shown that spin-orbit coupling does occur between these states which involves the promotion of a $\sigma$ $\left(p_{z}\right)$ electron from the ground state to a $\pi\left(p_{x}\right.$ or $\left.p_{y}\right)$ orbital in the excited state. However, insufficient information concerning the eigenvectors of the excited state wave functions is available to warrant a detailed $g$ shift analysis. Also, other excited states probably make significant contributions to the spin-orbit coupling and the value of $\Delta g_{1}$.

\section{DISCUSSION}

An interesting contrast between the properties of an isolated ion radical and a neutral radical in a solid neon matrix can be seen by the experimental results presented in Fig. 4. The laser vaporization of the SiB alloy also produces the $\mathrm{Si}_{2}^{+}$cation radical which has recently been studied by matrix ESR in our laboratory and found to have a ${ }^{4} \Sigma$ ground state. ${ }^{4}$ Visible light photolysis of the neon sample eliminates the ${ }^{28} \mathrm{Si}_{2}^{+}$and ${ }^{28,29} \mathrm{Si}_{2}^{+}$ESR lines while the neutral $\mathrm{SiAl}$ absorption is unaffected. The visible light is sufficiently energetic to ionize isolated anions; the liberated electrons can diffuse throughout the neon lattice and neutralize the various cation radicals. Unless the neutral radicals originally present undergo direct photochemical decomposition, which is not likely with visible light above $5000 \AA$, the response to photobleaching provides a most useful diagnostic tool in assigning the ESR spectra of isolated anions and cations. In this context, the negative response to photobleaching of the ESR features assigned to $\mathrm{SiAl}$ and $\mathrm{SiB}$ helps confirm the spectral assignments.

Using the laser vaporization approach, all attempts to observe the ESR spectrum of SiB or SiAl in argon matrices were unsuccessful. This observation suggests that the diatomic formation mechanism involves the codeposition reaction of ground or excited state atoms or ions during the condensation process, either on or just below the matrix surface. Atomic reactions are less likely to occur with argon matrices at $4 \mathrm{~K}$ because of reduced diffusion rates or greater quenching ability relative to ncon under similar deposition conditions. This negative ESR evidence indicates that neither $\mathrm{SiB}$ nor $\mathrm{SiAl}$ is a vapor phase species above their respective alloys under such energetic vaporization conditions.

Initially deposited argon matrices at $4 \mathrm{~K}$ containing $\mathrm{Si} / \mathrm{B}$ laser vaporization products showed no $\mathrm{H}_{2} \mathrm{BO}$ ESR absorptions. Upon warming the argon deposits to $28 \mathrm{~K}$ and recooling to $4 \mathrm{~K}$, intense $\mathrm{H}_{2} \mathrm{BO}$ ESR features appeared which consisted of a large isotropic hydrogen triplet [133.1(3) G] further split into smaller ${ }^{11} \mathrm{~B}$ quartets [30.6(2) G], and ${ }^{10} \mathrm{~B}$ septets [10.2(2) G]. The lowest and highest field components $\left(M_{J}= \pm 1\right)$ of the hydrogen triplet and associated boron hfs are shown in Fig. 7. Our argon magnetic parameter agree with those reported earlier by Graham and Weltner. ${ }^{34}$ Their argon formation conditions involved the reaction of boron atoms produced from a high temperature effusion oven, with impurities present in the matrix.

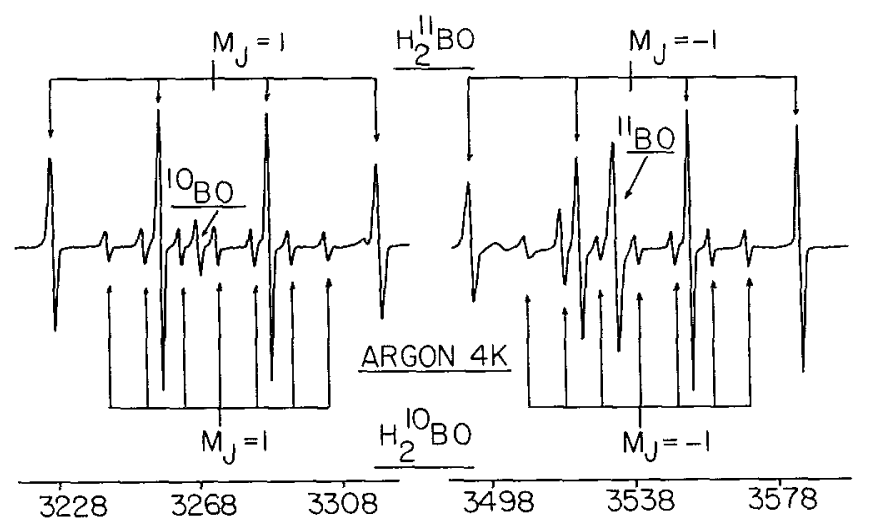

FIG. 7. Attempts to trap $\mathrm{SiB}$ in argon matrices were unsuccessful; however, intense $\mathrm{H}_{2} \mathrm{BO}$ ESR radical absorptions were observed in argon matrices after warming to approximately $30 \mathrm{~K}$ and quenching to $4 \mathrm{~K}$. The laser vaporization conditions employed for the argon experiments were similar to those used for the neon matrix experiments. The $\mathrm{H}_{2} \mathrm{BO}$ ESR spectrum consists of a large hydrogen triplet $(A=133 \mathrm{G})$ which is further split into a smaller ${ }^{11} \mathrm{~B}(I=3 / 2)$ quartet or a ${ }^{10} \mathrm{~B}(I=3)$ septet as labeled on the ESR spectrum for the lowest and highest transitions $\left(M_{J}= \pm 1\right)$ of the hydrogen triplet. The isotropic line shape probably indicates the oc. currence of motional averaging in the argon matrix.

Two interesting aspects of our observation of $\mathrm{H}_{2} \mathrm{BO}$ in these $\mathrm{Si} / \mathrm{B}$ laser deposition experiments are the previously unreported neon matrix magnetic parameters and the reaction mechanism involved at such low temperatures. Our neon ESR spectrum of $\mathrm{H}_{2} \mathrm{BO}$ was nearly isotropic with $A$ values of 129.1(4) $\mathrm{G}$ for hydrogen and 31.0(2) $\mathrm{G}$ for ${ }^{11} \mathrm{~B}$. While the smaller ${ }^{11} \mathrm{~B}$ parameters are equivalent within the stated experimental uncertainty in the two rare gas hosts, the hydrogen $A$ value is approximately $3 \%$ smaller in neon. Unlike argon, our initial neon deposits did exhibit the $\mathrm{H}_{2} \mathrm{BO}$ ESR signals which became only slightly more intense upon warming to $10 \mathrm{~K}$ and recooling to $4 \mathrm{~K}$. The greater and more rapid quenching ability of argon apparently prevented the $\mathrm{H}_{2} \mathrm{BO}$ formation reaction until the matrix was warmed. Hence, the diffusion dependent formation reaction seems to involve a reaction with an extremely small activation energy. We can only speculate about the identity of the argon reactants involved; possibilities include $\mathrm{H}+\mathrm{HBO}, 2 \mathrm{H}+\mathrm{BO}$, or $\mathrm{BH}+\mathrm{OH}$. The direct reaction of boron atoms with $\mathrm{H}_{2} \mathrm{O}$ does not seem likely under such low temperature conditions.

Given the importance of chemical vapor deposition (CVD) reactions of silicon compounds with diborane, $\mathrm{B}_{2} \mathrm{H}_{6}$, in the semiconductor industry for forming silicon doped materials, a series of neon trapping experiments was conducted to determine if SiB was formed when gaseous diborane was passed over silicon under laser vaporization conditions similar to those described in the experimental section. [See Fig. 1 of the $\mathrm{BNH}$ study involving $\mathrm{B}+\mathrm{NH}_{3}(\mathrm{~g})$ for more information on the experimental arrangement. $\left.{ }^{26}\right]$ No evidence of SiB formation was observed under these conditions; however, intense ESR absorptions for $\mathrm{SiH}_{3}$ and $\mathrm{HSiO}$ were detected. ${ }^{11,35}$ 


\section{ACKNOWLEDGMENTS}

Project support from the National Science Foundation (Grant No. CHE-9019511) and the Camille and Henry Dreyfus Foundation through their Scholar-Fellow program is gratefully acknowledged. Undergraduate student support was provided by a National Science FoundationResearch Experience for Undergraduates site grant and a Duke Endowment grant to Furman University. Equipment funds were made available from the Monsanto Foundation, the DuPont College Science Program, and the 3M Company. Appreciation is expressed to Dr. David Feller and Professor E. R. Davidson for use of their MELDF program for calculating the nuclear hyperfine parameters. M.D.M. and C.A.A. gratefully acknowledge research support from the National Science Foundation (Grant No. CHE8912673 ) and the donors of the Petroleum Research Fund.

\footnotetext{
'A. I. Boldyrev and J. Simons, J. Phys. Chem. (in press).

${ }^{2}$ MELDF was originally written by L. McMurchie, S. Elbert, S. Langhoff, and E. R. Davidson. It has been substantially modified by D. Feller, R. Cave, D. Rawlings, R. Frey, R. Daasch, L. Nitzche, P. Phillips, K. Iberle, C. Jackels, and E. R. Davidson.

${ }^{3}$ Lon B. Knight, Jr., S. T. Coranchi, and E. Earl, J. Chem. Phys. 88, 7348 (1988).

${ }^{4}$ Lon B. Knight, Jr., J. O. Herlong, Robert Babb, E. Earl, Devon W, Hill, and C. A. Arrington, J. Phys. Chem. 95, 2732 (1991).

${ }^{5}$ Lon B. Knight, Jr. (unpublished). The electronic ground state of $\mathrm{Sn}_{2}^{+}$ was determined to be $X^{4} \Sigma$ while our ESR results indicate the $\mathrm{Pb}_{2}^{+}$does not have a ${ }^{4} \Sigma$ ground state.

'Lon B. Knight, Jr., S. T. Cobrnachi, J. T. Petty, E. Earl, David Feller, and E. R. Davidson, J. Chem. Phys. 90, 690 (1989).

${ }^{7}$ Lon B. Knight, Jr., Scott T. Cobranchi, John O. Herlong, and C. A. Arrington, J. Chem. Phys. 92, 5856 (1990).

${ }^{8}$ Lon B. Knight, Jr. and John O. Herlong, J. Chem. Phys. 91, 69 (1989).

${ }^{\circ}$ Lon B. Knight, Jr, and J. T. Petty, J. Chem. Phys. 88, 481 (1988).

${ }^{10}$ Lon B. Knight, Jr., Robert M. Babb, Gina M. King, Allan J. McKinley, Michael D. Morse, and Caleb A. Arrington, J. Chem. Phys. (in press).

"W. Weltner, Jr., Magnetic Atoms and Molecules (Dover, Mineola, NY, 1989).

${ }^{12}$ R. J. Van Zee, S. Li, Y. M. Hamrick, and W. Weltner, Jr., J. Chem. Phys. (in press).
}

${ }^{13}$ Lon B. Knight, Jr., in Chemistry and Physics of Matrix-Isolated Species, edited by L. Andrews and M. Moskovits (North-Holland, Amsterdam, 1989), Chap. 7.

${ }^{14}$ Lon B. Knight, Jr., in Radical Ionic Systems, edited by A. Lund and M. Shiotani (Kluwer Academic, Dordrecht, The Netherlands, 1991), pp. 73-97.

${ }^{15}$ Lon B. Knight, Jr., Acc. Chem. Res. 19, 313 (1986).

${ }^{16}$ P. H. Kasai, J. Am. Chem. Soc. 113, 1539 (1991); 112, 4313 (1990).

${ }^{17}$ A. J. McKinley and J. Michl, J. Phys. Chem. 95, 2674 (1991).

${ }^{18}$ Lon B. Knight, Jr., Kelly Kerr, Martha Villanueva, Allan J. McKinley, and David Feller, J. Chem. Phys. 97, 5363 (1992).

${ }^{19} \mathrm{~T}$. Bally, in Radical Ionic Systems, edited by A. Lund and M. Shiotani (Kluwer Academic, Dordrecht, 1991), pp. 3-52; T. Shida, E. Haselback, and T. Bally, Acc. Chem. Res. 17, 180 (1984); K. Toriyama, K. Nunome, and M. Isasaki, J. Chem. Phys. 77, 5891 (1982).

${ }^{20}$ F. Shimura, Semiconductors Silicon Crystal Technology (Academic, Boston, 1989).

${ }^{21}$ G. Verhaegen, F. E. Stafford, M. Ackerman, and J. Drowart, Nature (London) 193, 1280 (1962); G. Verhaegen, F. E. Stafford, and J. Drowart, J. Chem. Phys. 40, 1622 (1962).

${ }^{22}$ T. Goto, M. Mukaida, and T. Hirai, Mater. Res. Soc. Symp. Proc. 168, 167 (1990); A. R. Nicoll, U. W. Hildebrandt, and G. Wahl, Thin Solid Films 64, 321 (1979).

${ }^{23}$ C. A. Stearns and F. J. Kohl, High Temp. Sci. 5, 113 (1973).

${ }^{24}$ Lon B. Knight, Jr., Robert Babb, Devon W. Hill, and Allan J. McKinley, J. Chem. Phys. 97, 2987 (1992).

${ }^{25}$ Lon B. Knight, Jr., B. W. Gregory, S. T. Cobranchi, David Feller, and E. R. Davidson, J. Am Chem. Soc. 109, 3521 (1987).

${ }^{26}$ Lon B. Knight, Jr., John O. Herlong, Thomas J. Kirk, and C. A. Arrington, J. Chem. Phys. 96, 5604 (1992).

${ }^{27}$ Lon B. Knight, Jr. and J. Steadman, J. Chem. Phys. 78, 5940 (1983).

${ }^{28}$ M. Iwasaki, J. Magn. Res. Reson. 16, 417 (1974).

${ }^{29}$ K. P. Huber and G. Herzberz, Molecular Spectra and Molecular Structure. IV. Constants of Diatomic Molecules (Van Nostrand Reinhold, New York, 1979).

${ }^{30}$ H. Hotop and W. C. Lineberger, J. Chem. Phys. 14, 731 (1985).

${ }^{3 \mathrm{l}}$ T. H. Dunning, Jr. and P. J. Hay, Methods of Electronic Structure Theory, edited by H. F. Schaefer III (Plenum, 1977).

${ }^{32}$ J. R. Morton and K. F. Preston, J. Magn. Reson. 30, 577 (1978).

${ }^{33}$ Lon B. Knight, Jr., A. Ligon, R. W. Woodward, David Feller, and E. R. Davidson, J. Am. Chem. Soc. 107, 2857 (1985).

${ }^{34}$ W. R. M. Graham and W. Weltner, Jr., J. Chem. Phys. 65, 1516 (1976).

${ }^{35}$ R. J. Van Zee, R. F. Ferrante, and W. Weltner, Jr., J. Chem. Phys. 83, 6181 (1985). 